\title{
Factors and prevalence of tooth erosion in children
}

\author{
The prevalence of tooth erosion in 12-year-old children and A multifactorial analysis of factors associated with \\ dental erosion C. R. Dugmore and W. P. Rock Br Dent J 2004; 196: 279-282; 283-286
}

\section{The prevalence of tooth erosion in 12-year-old children}

\section{Objectives}

To establish the prevalence of tooth erosion in a representative sample of 12-year-old children in Leicestershire and Rutland. To determine if gender, ethnic group, deprivation or caries experience influences the prevalence of erosion.

Methods

A random sample of 1,753 12-year-olds resident in Leicestershire and Rutland were examined in 62 schools; 906 were boys and 847 girls; 1,379 were Caucasian and 316 Asian. Tooth erosion was assessed using the index employed in the survey of Children's Dental Health in England and Wales (1993). The Townsend index was used to record deprivation.

Results

Tooth erosion was found in 59.7\% of the children, with $2.7 \%$ exhibiting exposed dentine. Significantly more boys than girls; Caucasian than Asian children; and those with caries experience, had erosion present (chi-square for all $\mathrm{P}<0.01$ ). Overall no significant difference was found between deprivation categories, however socio-economically advantaged Caucasian children had significantly less tooth erosion than other groups.

Conclusions

There was a high prevalence of tooth erosion in 12-year-old children. Significantly more erosion occurred in boys than girls, and culture appeared to influence prevalence. Children with caries experience had a higher prevalence of erosion than those without caries, which may reflect a lower level of dietary care. Deprivation seemed to affect the prevalence of tooth erosion in Caucasian children.

\section{COMMENT}

These two papers present data which adds significantly to our dental erosion knowledge. Problems exist in the standardized measurement criteria for erosion - effectively an examiner can determine that the erosive process has started (loss of surface characteristics) but the next visibly determinable stage is exposure of dentine (ie loss of total enamel thickness). This crude visual assessment limits the usefulness of these criteria in terms of determination of aetiological factors. However, by using standardized criteria and an experienced calibrated examiner on a large sample size, the authors have demonstrated some very important aspects of erosion in 12-year-olds and a number of associated factors in 12- and 14-year-olds.

Firstly, they confirm previous recent findings of a high prevalence of enamel erosion in this age group. After only six years in the mouth, teeth in over half of this sample of 12-year-olds showed visually detectable evidence of erosion. Given the prevalence figures collected in the early 1990s and that collected in the last five years, this study adds yet more evidence to the data demonstrating that erosion in adolescents has dramatically increased in prevalence in the last decade.

The authors' tabulation of all previous UK data on erosion in

\section{A multifactorial analysis of factors associated with dental erosion}

\section{Objectives}

This study prospectively examines the relationship of possible aetiological factors to the presence of tooth erosion in a cohort of children.

Patients and methods

A random sample of 1,753 children was examined at age 12 and 1,308 of the same children were re-examined at age 14 years. The children were asked to complete questionnaires on both occasions, 1,149 subjects gave usable replies. The erosion index used was based upon the 1993 Survey of Children's Dental Health. Results were analysed using logistic regression.

Results

At age 12 significant positive associations were found between erosion and decay experience (odds ratio $[\mathrm{OR}]=1.48$ ), drinking fruit juice $(\mathrm{OR}=1.42$ ) or fizzy pop $(\mathrm{OR}=1.59-2.52$, depending on amount and frequency). The presence of calculus (OR 0.48) or eating fruit other than apples or citrus fruit (OR 0.48) reduced the chances of erosion. High consumption of carbonated drinks increased the odds of erosion being present at 12 years by $252 \%$ and was a strong predictor of the amount of erosion found at age 14 .

Conclusions

Of the factors investigated, a history of dental caries and a high consumption of carbonated drinks were most closely related to the presence of dental erosion. The risk of erosion bore a strong relationship to the amount and frequency of carbonated drink consumption.

children is extremely useful. The new data on ethnic minorities is important and may help to elucidate aetiological factors. The powerful associations between the presence of erosion and the risk of erosion in these samples are striking. The consumption of carbonated drinks was clearly significantly associated with erosion in this large longitudinal cohort study. Since there was a significant association between the increasing amount and frequency of carbonated drink consumption and the increased risk of erosion this appears to represent powerful evidence for an aetiological relationship. The development of technologies to accurately measure and monitor erosion at a sub-clinical level, which appears to be not too far away, will build on this current epidemio-logical work and help determine if (and the extent to which) such an aetiological relationship exists.

With over 90\% of 14-year-olds in this sample experiencing erosion, this is a clinical problem which might best be described as a 'slow-release time-bomb' of immense proportions. The data collected represent a significant 'brick in the wall' of evidence in the unfolding of our understanding of erosion.

Dr Chris Longbottom, Senior Lecturer in Preventive \& Children's Dentistry, Dundee Dental School, University of Dundee doi:10.1038/sj.bdj.4811038 